\title{
On the Stable Throughput of Cooperative Cognitive Radio Networks with Finite Relaying Buffer
}

\author{
Adel M. Elmahdy ${ }^{\dagger}$, Amr El-Keyi ${ }^{\dagger}$, Tamer ElBatt ${ }^{\dagger}$ and Karim G. Seddik* \\ ${ }^{\dagger}$ Wireless Intelligent Networks Center (WINC), Nile University, Giza, Egypt. \\ ${ }^{\S}$ EECE Dept., Faculty of Engineering, Cairo University, Giza, Egypt. \\ *Electronics Engineering Department, American University in Cairo, AUC Avenue, New Cairo 11835, Egypt. \\ Email: adel.elmahdy@ieee.org, aelkeyi@nileuniversity.edu.eg, telbatt@ieee.org, kseddik@aucegypt.edu
}

\begin{abstract}
In this paper, we study the problem of cooperative communications in cognitive radio systems where the secondary user has limited relaying room for the overheard primary packets. More specifically, we characterize the stable throughput region of a cognitive radio network with a finite relaying buffer at the secondary user. Towards this objective, we formulate a constrained optimization problem for maximizing the secondary user throughput while guaranteeing the stability of the primary user queue. We consider a general cooperation policy where the packet admission and queue selection probabilities, at the secondary user, are both dependent on the state (length) of the finite relaying buffer. Despite the sheer complexity of the optimization problem, attributed to its non-convexity, we transform it to a linear program. Our numerical results reveal a number of valuable insights, e.g., it is always mutually beneficial to cooperate in delivering the primary packets in terms of expanding the stable throughput region. In addition, the stable throughput region of the system, compared to the case of infinite relaying queue capacity, marginally shrinks for limited relaying queue capacity.

Index Terms-Wireless networks, cognitive radio, cooperative communications, stable throughput region, convex optimization.
\end{abstract}

\section{INTRODUCTION}

The concept of cognitive radios was motivated by the problem of spectrum scarcity, as well as the inefficient utilization of the licensed spectrum [1], [2]. The fundamental purpose of deploying cognitive radio networks is to enhance the spectrum utilization by exploiting spectral holes where a portion of the licensed spectrum is unutilized for a period of time. Accordingly, the cognitive radio technology proposes the coexistence of unlicensed secondary users (SUs) with the licensed primary users (PUs) on the same frequency band in such a way that a minimum quality of service (QoS) is guaranteed for the PUs. For example, in overlay cognitive radio networks, the $\mathrm{SU}$ seizes the opportunity to transmit its packets when it senses a spectrum hole, that is, the PU is idle.

In wireless networks, the notion of cooperative communications has attracted considerable attention in recent years. The basic idea relies on the broadcast nature of the wireless

This work was made possible by grants number NPRP 4-1034-2-385 and NPRP 5-782-2-322 from the Qatar National Research Fund (a member of Qatar Foundation). The statements made herein are solely the responsibility of the authors. channel where intermediate nodes between the transmitter and receiver can be thought of as potential relay nodes. If a receiver fails to decode a packet, there is a good chance that one of the intermediate nodes has received this packet and, hence, can aid in delivering it to the receiver. Therefore, cooperative communications can play a major role in enhancing the performance of wireless networks. Cooperative communications has been explored within the framework of cognitive radio networks whereby the SU helps the PU in delivering its packets to the destination [3], [4]. Intuitively, both the PU and the SU benefit from such cooperation. The PU reliably delivers its own packets while the SU witnesses an increase in the number of time slots available for transmission.

A considerable part of the wireless literature was dedicated to the concept of cooperative communications from the perspective of the physical layer, e.g., [3], [4], [5]. Others studied it from the perspective of the MAC layer, e.g., [6], [7]. Our prime focus in this paper is on cooperative relaying in cognitive radio networks at the packet level. Essentially, we extend the work presented in [8], [9] and [10]. In [8], a new protocol-level full cooperation in a wireless multiple-access system is proposed for a system composed of $N$ users in which each node is a source, and at the same time a potential relay. In [9], [10], the authors propose a cooperative strategy with probabilistic relaying. In this strategy, it is considered that the SU has two infinite-length queues; one is for its own packets and the other is for relaying the PU packets. Upon overhearing a PU transmission, the SU enqueues the PU packet, if not correctly decoded by the destination, with probability (w.p.) $a$. Conversely, when the PU is sensed idle, the SU serves its own data queue w.p. $b$ or the relaying queue w.p. $1-b$.

Our main contribution in this paper is to characterize the stable throughput region of the system in [10] when the relaying buffer at the SU has finite capacity. This, in turn, renders the problem more challenging due to the added complexity of modelling the state of the SU relay queue and solving the associated optimization problem. The motivation behind our work is to investigate the case when the SU helps the PU in delivering its packets, yet, with limited resources, as opposed to unlimited resources in [8], [10]. It can be contemplated that the proposed system model constitutes an important step towards real systems. Furthermore, we extend the system degrees of 


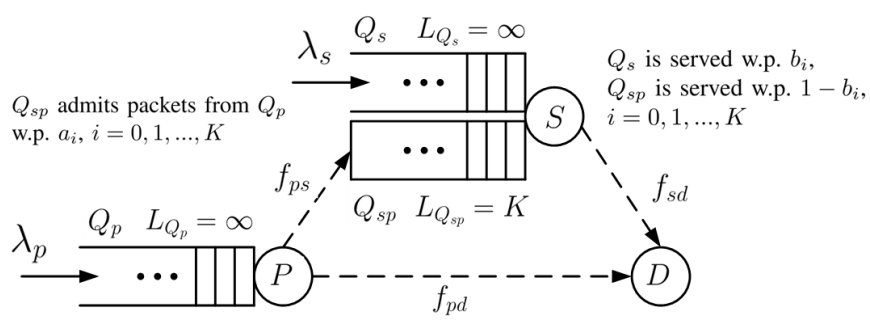

Fig. 1. The system model.

freedom whereby the probability of the SU enqueueing an overheard PU packet is dependent on the state of the relaying queue, i.e., relaying queue length at a given time slot. In addition, the SU queue selection probabilities, between the relay queue and its own packets queue, is also assumed to be state-dependent. This problem formulation yields a nonconvex optimization problem. Nevertheless, we exploit the structure of the problem that enables us to transform it into a linear program that can be efficiently solved. We study the effect of the finite size of the relaying queue on the stable throughput region of the system. The numerical results show that the stable throughput region, compared to the case of infinite relaying buffer size, marginally shrinks for relatively small relaying queue lengths.

The rest of this paper is organized as follows. In Section II, the system model is described. Section III demonstrates the proposed cooperation policy. The characterization of the stable throughput region of the system as an optimization problem is investigated in Section IV. Numerical results are presented in Section V. Finally, Section VI concludes the work and points out potential directions for future research.

\section{SYSTEM MODEL}

We consider the cooperative cognitive radio network shown in Fig. 1. The network consists of a PU, an SU, and a common destination. The PU is equipped with an infinite queue $\left(Q_{p}\right)$ for its own packets. On the other hand, the SU is equipped with an infinite queue $\left(Q_{s}\right)$ for its own packets and a finite queue $\left(Q_{s p}\right)$ of size $K$ for the packets overheard, decoded and enqueued from the PU. The system is time-slotted where the packet transmission from either user to the destination takes precisely one time slot. The external packet arrivals at $Q_{p}$ and $Q_{s}$ are modelled as Bernoulli random processes with average arrival rates $\lambda_{p}$ and $\lambda_{s}$ packets per slots, respectively, where $0 \leq$ $\lambda_{p}, \lambda_{s} \leq 1$. The arrival processes of both users are assumed to be independent from each other across time slots. Following the notation in [10], The instantaneous length evolution of an arbitrary queue $i$ is defined as

$$
Q_{i}^{t+1}=\left(Q_{i}^{t}-Y_{i}^{t}\right)^{+}+X_{i}^{t}, i \in\{p, s, s p\}
$$

where $Q_{i}^{t}$ is the number of packets at the beginning of time slot $t$, and $X_{i}^{t}$ and $Y_{i}^{t}$ are the number of arriving and departing packets in time slot $t$, respectively. The random variables, $X_{i}^{t}$ and $Y_{i}^{t}$, are binary taking values 0 or 1 . Note that $(Z)^{+}=$ $\max (Z, 0)$.
The quality of a wireless communication channel is deteriorated due to the inherent impairments caused by signal attenuation, shadowing, multipath fading, and additive noise. The quality of the channel is abstracted in this work by the average channel reception probability which is the probability that a transmitted packet is correctly decoded. It is assumed that the SU has a perfect sensing capability and, hence, at most one of the two users will transmit in every time slot. Consequently, the only reason for packet loss is link outage. Since we assume that the channel gain and noise processes are stationary, and the mobility of the nodes is ignored in our analysis, the channel success probabilities are deterministic and take values from the interval $[0,1]$. Let $f_{p d}, f_{p s}$ and $f_{s d}$ denote the probability of successful packet reception from the PU to the destination, from the PU to the SU, and from the SU to the destination, respectively. Throughout our analysis, we assume that $f_{p d}<f_{s d}$, i.e., the $\mathrm{SU}$ has a better channel to the destination than the PU. We will signify the prominence of such assumption to the cooperation strategy later.

Acknowledgement packets (ACKs) are sent either by the destination for successfully decoded packets from the PU or SU, or by the SU for successfully-decoded overheard primary packets. We assume that ACKs are instantaneous as well as error-free. Additionally, ACKs can be heard by all the nodes in the system.

\section{CoOperation With Finite RELAying BUfFer AT THE SU}

The proposed system adopts the following cooperative policy at the MAC layer. When the PU is backlogged, i.e., $Q_{p}$ is non-empty, it transmits a packet. In such case, we have three possible scenarios. If the destination correctly decodes the PU packet, it sends back an ACK that is heard by the PU and the packet is then dropped from $Q_{p}$ and exits the system. On the other hand, if the destination fails to decode the PU packet, but the SU correctly decodes it, $Q_{s p}$ buffers the packet w.p. $a_{i}, i=0,1, \ldots, K$. The packet admission probability in the system depends on the number of packets in $Q_{s p}$. If the SU admits the packet, it sends back an ACK so that the PU drops the packet from its queue. Therefore, the SU is in charge of transmitting this packet to the destination. Finally, if neither the destination nor the SU successfully receives the PU packet, the PU keeps this packet in its queue to be retransmitted in the next time slot.

When the PU is idle, i.e., $Q_{p}$ is empty, the $\mathrm{SU}$ accesses the channel. It transmits a packet either from $Q_{s p}$ w.p. $1-b_{i}, i=$ $0,1, \ldots, K$ or from $Q_{s}$ w.p. $b_{i}, i=0,1, \ldots, K$. The queue selection probability in the system also depends on the number of packets in $Q_{s p}$. If the destination correctly decodes the SU packet, it sends back an ACK that is heard by the SU. The packet is then dropped from either $Q_{s p}$ or $Q_{s}$ and exits the system.

In view of the finite queue size of $Q_{s p}$, we should account for the blocked packets when the relaying queue is full at a given time slot, i.e., when it has $K$ packets. This case is handled by forcing the system to not accept any relayed 
packets from the PU when $Q_{s p}$ is full. In other words, we set $a_{K}=0$. On the other hand, a time slot would be wasted if an empty $Q_{s p}$ is selected for transmission while $Q_{s}$ still has packets to transmit. In order to prevent such case, the system is forced to select $Q_{s}$ for transmission whenever $Q_{s p}$ is empty, In other words, $b_{0}=1$.

According to the aforementioned cooperation policy, the system at hand is non-work-conserving because there are cases where the system might waste time slots in spite of having packets to transmit. A typical case occurs when an empty $Q_{s}$ is probabilistically selected for transmission while $Q_{s p}$ still has packets to transmit. Thus, the system would waste such time slots. Nonetheless, the non-work-conserving policy of our system achieves the same stable throughput region of the work-conserving policy characterized in [8] using an infinite relaying buffer. This result stems from optimally tuning the system degrees of freedom provided by the probabilistic packet admission and queue selection of the proposed cooperation policy with finite relaying buffer. The optimization problem and performance results supporting the above insights will follow in the next two sections.

\section{The Stable Throughput Region}

\section{A. Quеиe Stability}

In a stable network of queues, every individual queue has to be stable. Loynes' theorem establishes the condition for stability of an infinite size queue [11]. It asserts that if the queue arrival and service processes are stationary, the queue is stable if and only if the packet arrival rate $\lambda$ is strictly less than the packet service rate $\mu$. It is worth mentioning that $Q_{s p}$, with maximum size $K$, is stable for all positive values of the arrival and service rates. The number of packets will never grow to infinity since it is upper bounded by $K$. Let $\pi_{i}$, $i=0,1, \ldots, K$, be defined as the probability that $Q_{s p}$ is in a given state $i$, i.e., $Q_{s p}$ has $i$ packets, at a given time slot.

Note that a packet leaves $Q_{p}$ if it is either correctly decoded by the destination or correctly decoded by the SU and admitted to the relaying queue. Therefore,

$$
\mu_{p}=f_{p d}+\left(1-f_{p d}\right) f_{p s} \sum_{i=0}^{K} a_{i} \pi_{i}
$$

Also, a packet leaves $Q_{s}$ if the PU is idle, $Q_{s}$ is selected for transmission, and the packet is correctly decoded by the destination. Therefore,

$$
\mu_{s}=f_{s d}\left(1-\frac{\lambda_{p}}{\mu_{p}}\right) \sum_{i=0}^{K} b_{i} \pi_{i}
$$

Accordingly, the stable throughput region of the system is characterized as

$$
\begin{aligned}
\mathcal{R}=\left\{\left(\lambda_{p}, \lambda_{s}\right) \mid \lambda_{s}<f_{s d}\left(1-\frac{\lambda_{p}}{\mu_{p}}\right) \sum_{i=0}^{K} b_{i} \pi_{i}\right. \\
\text { for } \left.\lambda_{p}<f_{p d}+\left(1-f_{p d}\right) f_{p s} \sum_{i=0}^{K} a_{i} \pi_{i}\right\}
\end{aligned}
$$

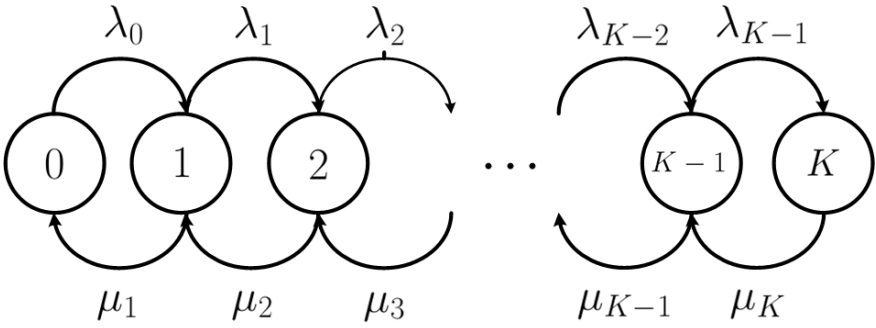

Fig. 2. Discrete Time Markov Chain (DTMC) model of the SU relaying queue, $Q_{s p}$.

In pursuance of completely characterizing the stable throughput region of the system, the steady-state distribution of $Q_{s p}$ should be calculated. In the next subsection, we conduct a Discrete Time Markov Chain (DTMC) analysis for $Q_{s p}$.

\section{B. DTMC Analysis of $Q_{s p}$}

Fig. 2 depicts the state transition diagram of $Q_{s p}$ where state $i$ denotes the number of packets in $Q_{s p}$. The arrival rate $\lambda_{i}$ can be characterized as the conditional probability that $Q_{p}$ is non-empty, the destination fails to decode the PU packet, the SU correctly decodes that packet, and $Q_{s p}$ admits the relayed packet, given that $Q_{s p}$ is at state $i$. Thus,

$$
\lambda_{i}=\frac{\lambda_{p}}{\mu_{p}}\left(1-f_{p d}\right) f_{p s} a_{i}, i=0,1, \ldots, K
$$

Similarly, $\mu_{i}$ can be characterized as the conditional probability that $Q_{p}$ is idle, the SU selects $Q_{s p}$ to transmit the enqueued PU packet, and the destination correctly decodes it, given that $Q_{s p}$ is at state $i$. Thus,

$$
\mu_{i}=\left(1-\frac{\lambda_{p}}{\mu_{p}}\right)\left(1-b_{i}\right) f_{s d}, i=0,1, \ldots, K
$$

It should be noted that $\lambda_{K}=0$ as $a_{K}=0$ and $\mu_{0}=0$ as $b_{0}=1$.

Using the balance equations, the steady-state probabilities can be expressed as

$$
\pi_{j+1}=\frac{\lambda_{j}}{\mu_{j+1}} \pi_{j}
$$

where $j=0,1, \ldots, K-1$. Substituting from (5) and (6) in (7), we get

$\pi_{j+1}=\frac{\lambda_{p}\left(1-f_{p d}\right) f_{p s} a_{j}}{\left(\mu_{p}-\lambda_{p}\right)\left(1-b_{j+1}\right) f_{s d}} \pi_{j} \quad$ for $j=0,1, \ldots, K-1$

Applying the normalization condition,

$$
\sum_{i=0}^{K} \pi_{i}=1
$$

along with (8), one can obtain the value of $\pi_{0}$ and, hence, completely characterize the steady-state distribution of $Q_{s p}$. 


\section{Stable throughput characterization problem}

Taking into consideration our foregoing discussion in the previous two subsections, the problem of defining the stable throughput region of the system can be formulated as maximizing the service rate of the SU for a given arrival rate of the primary traffic under stability constraints on all queues in the system, i.e.,

$$
\begin{aligned}
\max _{\mu_{p},\left\{a_{i}, b_{i}, \pi_{i}\right\}} & f_{s d}\left(1-\frac{\lambda_{p}}{\mu_{p}}\right) \sum_{i=0}^{K} b_{i} \pi_{i} \\
\text { s. t. } & 0 \leq a_{i}, b_{i}, \pi_{i} \leq 1 \quad \text { for } i=0,1, \ldots, K \\
& a_{K}=0, b_{0}=1 \\
& \mu_{p}=f_{p d}+\left(1-f_{p d}\right) f_{p s} \sum_{i=0}^{K} a_{i} \pi_{i} \\
& \lambda_{p}<\mu_{p} \\
& 89
\end{aligned}
$$

Note that the optimization problem in 10$]$ is non-convex. Nevertheless, we exploit the structure of the problem to transform it into a linear program as follows.

First, we introduce the optimization variables $x_{i}=a_{i} \pi_{i}$, and $y_{i}=b_{i} \pi_{i}$, where $i=0,1, \ldots, K$. Therefore, we can write (2) as

$$
\mu_{p}=f_{p d}+\left(1-f_{p d}\right) f_{p s} \sum_{i=0}^{K} x_{i}
$$

Since $a_{i}, b_{i}, \pi_{i} \in[0,1]$, for $i=0,1, \ldots, K$, we have the following constraints on $x_{i}$ and $y_{i}$,

$$
\begin{gathered}
0 \leq x_{i}, y_{i} \leq \pi_{i} \quad \text { for } i=0, \ldots, K \\
0 \leq \sum_{i=0}^{K} x_{i} \leq 1,0 \leq \sum_{i=0}^{K} y_{i} \leq 1
\end{gathered}
$$

Furthermore, we can write the objective function of $(10)$ and the constraint in (8) as

$$
\begin{gathered}
\mu_{s}=f_{s d}\left(1-\frac{\lambda_{p}}{\mu_{p}}\right) \sum_{i=0}^{K} y_{i} \\
\left(\pi_{j+1}-y_{j+1}\right)\left(\mu_{p}-\lambda_{p}\right)=\frac{\lambda_{p}}{f_{s d}}\left(1-f_{p d}\right) f_{p s} x_{j} \\
\text { for } j=0, \ldots, K-1
\end{gathered}
$$

Next, we rewrite the optimization problem in 10 . The resulting problem is given by

$$
\begin{aligned}
\max _{\mu_{p},\left\{x_{i}, y_{i}, \pi_{i}\right\}} & f_{s d}\left(1-\frac{\lambda_{p}}{\mu_{p}}\right) \sum_{i=0}^{K} y_{i} \\
\text { s. t. } & 0 \leq \pi_{i} \leq 1 \quad \text { for } i=0,1, \ldots, K \\
& x_{K}=0, y_{0}=\pi_{0} \\
& \mu_{p}=f_{p d}+\left(1-f_{p d}\right) f_{p s} \sum_{i=0}^{K} x_{i} \\
& \lambda_{p}<\mu_{p} \\
& \text { 91 }, \text { (12), (13) }
\end{aligned}
$$

The above optimization problem is still non-convex. However, at a given value of $\mu_{p}$, the problem reduces into a linear program in the variables $\left\{a_{i}, b_{i}, \pi_{i}\right\}$ which is given by

$$
\begin{aligned}
\max _{\left\{x_{i}, y_{i}, \pi_{i}\right\}} & f_{s d}\left(1-\frac{\lambda_{p}}{\mu_{p}}\right) \sum_{i=0}^{K} y_{i} \\
\text { s. t. } & 0 \leq \pi_{i} \leq 1 \quad \text { for } i=0,1, \ldots, K \\
& x_{K}=0, y_{0}=\pi_{0} \\
& 9,12,13,15
\end{aligned}
$$

It can be evidently shown from (2) and (4) that the feasible values of $\mu_{p}$ over which the linear program runs is

$$
\max \left(\lambda_{p}, f_{p d}\right) \leq \mu_{p} \leq f_{p d}+\left(1-f_{p d}\right) f_{p s}
$$

To recapitulate briefly, we transformed the non-convex optimization problem of finding the stable throughput region of the system into a linear program via standard techniques. At a given throughput of the PU, we run the resulting linear program in (17) under the feasible values of $\mu_{p}$ given by (18). Our goal is to identify the value of $\mu_{p}$ that corresponds to the maximum achievable value of the objective function, i.e., the service rate of the SU, while satisfying the system constraints.

In the next section, we solve the aforementioned linear program. The numerical results will reveal the underlying trend of the optimal solution of the problem.

\section{Numerical Results}

In this section, we conduct a performance evaluation for the system under the proposed cooperative policy. The system parameters are the probabilities of no link outages between the different nodes that are selected to be $f_{p d}=0.3, f_{p s}=0.4$, $f_{s d}=0.8$. Moreover, a baseline comparison with the workconserving scheme in which the SU fully cooperates with the PU via an infinite length queue [8] is presented. Furthermore, we used CVX, a package for solving convex optimization programs [12], [13], in order to solve the linear program in (17).

Fig. 3 depicts the stable throughput region of the system for different lengths of the relaying queue $Q_{s p}$. The lower bound of the stable throughput region occurs when $K=0$. This corresponds to 'No Cooperation' scenario whereby the SU doesn't cooperate with the PU in delivering its packets. On the other hand, the upper bound of the stable throughput region occurs when $K=200$, i.e., the queue length is relatively long with respect to the system parameters. This corresponds to 'Full Cooperation, Infinite Queue Length' scenario [8]. Furthermore, As the queue length varies from $K=0$ to $K=200$, the stable throughput region increases as shown in Fig. 3. In other words, on the assumption that $f_{s d} \geq f_{p d}$, when the level of cooperation in delivering the PU packets raises by increasing the length of the relaying queue $Q_{s p}$, the likelihood that $Q_{p}$ is empty increases and the $\mathrm{SU}$ can safely inject more packets into $Q_{s}$ without violating the system constraints. As a result, a corresponding increase in the throughput of the $\mathrm{SU}$ takes place. Moreover, it is obvious that the decrease in the stable throughput region, compared to the case of infinite 


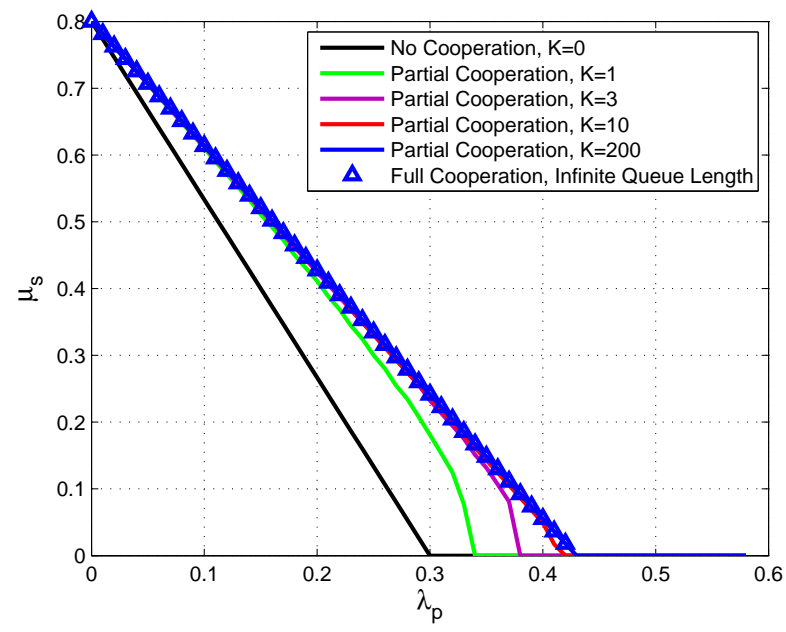

Fig. 3. The stable throughput regions of the system under different cooperative and non-cooperative schemes for different lengths of the relaying queue $Q_{s p}\left(f_{p d}=0.3, f_{p s}=0.4\right.$ and $\left.f_{s d}=0.8\right)$.

relaying buffer size, is marginal even for relaying buffer sizes as small as 10 packets. In other words, the system doesn't lose much despite the limited relaying room.

Investigating the solution of our constrained optimization problem of maximizing the SU throughput for a specific queue length $K$, one can identify the underlying trend of the optimal solution of the probability of selecting $Q_{s p}, 1-b_{i}$, where $i=1,2, \ldots, K$. As the PU injects more packets to the system, i.e., $\lambda_{p}$ increases, the probability of selecting $Q_{s p}, 1-b_{i}$, $i=1,2, \ldots, K$, increases. This is equivalent to giving more priority to $Q_{s p}$ over $Q_{s}$ so as to accommodate the increase in $\lambda_{p}$. This is a way to explains why $\lambda_{s}$ is inversely proportional to $\lambda_{p}$. In addition to this partial cooperation solution, the full cooperation solution, $1-b_{i}=1, i=1,2, \ldots, K$, also leads to the same maximum achievable stable throughput region. Our solution of the optimization problem exploits the introduced degree of freedom of the formulated problem that is represented in the dependency of the queue selection probability on $Q_{s p}$ state at every time slot. Therefore, at every value of $\lambda_{p}$, the solver assigns the minimum $1-b_{i}$ that maximize the objective function while satisfying all the system constraints. However, the full cooperation solution assigns a fixed value for $1-b_{i}$ regardless of the value of $\lambda_{p}$.

\section{CONCLUSION}

We study the stable throughput region of a cooperative cognitive radio network when the relaying buffer at the SU has a finite capacity. We demonstrate a rigorous mathematical formulation for the problem. Although the formulation results in a constrained non-convex optimization problem, the problem is reformulated, via standard techniques, to be a linear program. Numerical results reveal the fact that cooperation of the SU in delivering the packets of the PU is always advantageous to both users in terms of expanding their stable throughput region. Furthermore, the numerical results show that the sys- tem does not lose much in terms of the stable throughput region despite the limited relaying capacity. Finally, solving the optimization problem yields a partial cooperation solution, in addition to the full cooperation solution, that maximizes the service rate of the SU due to the degrees of freedom introduced in the packet admission and queue selection probabilities. This is embodied in the dependency of such probabilities on the number of packets in the relaying queue. For future work, other performance metrics of the network can be studied in the light of the new dimensions added to the problem formulation.

\section{REFERENCES}

[1] J. Mitola III, "Cognitive radio: An integrated agent architecture for software defined radio," PhD dissertation, Royal Institute of Technology (KTH), pp. 271-350, May 2000.

[2] S. Haykin, "Cognitive radio: brain-empowered wireless communications," IEEE Journal on Selected Areas in Communications, vol. 23, no. 2, pp. 201-220, Feb. 2005.

[3] J. N. Laneman, D. N. C. Tse, and G. W. Wornell, "Cooperative diversity in wireless networks: Efficient protocols and outage behavior," IEEE Transactions on Information Theory, vol. 50, no. 12, pp. 3062-3080, Dec. 2004.

[4] G. Kramer, M. Gastpar, and P. Gupta, "Cooperative strategies and capacity theorems for relay networks," IEEE Transactions on Information Theory, vol. 51, no. 9, pp. 3037-3063, Sept. 2005.

[5] K. Azarian, H. El Gamal, and P. Schniter, "On the achievable diversitymultiplexing tradeoff in half-duplex cooperative channels," IEEE Transactions on Information Theory, vol. 51, no. 12, pp. 4152-4172, Dec. 2005.

[6] A. K. Sadek, K. J. R. Liu, and A. Ephremides, "Cognitive multiple access via cooperation: protocol design and performance analysis," IEEE Transactions on Information Theory, vol. 53, no. 10, pp. 3677-3696, Oct. 2007.

[7] I. Krikidis, J. N. Laneman, J. S. Thompson, and S. McLaughlin, "Protocol design and throughput analysis for multi-user cognitive cooperative systems," IEEE Transactions on Wireless Communications, vol. 8, no. 9, pp. 4740-4751, Sept. 2009.

[8] B. Rong and A. Ephremides, "Cooperative access in wireless networks: stable throughput and delay," IEEE Transactions on Information Theory, vol. 58, no. 9, pp. 5890-5907, Sept. 2012.

[9] M. Ashour, A. A. El-Sherif, T. ElBatt, and A. Mohamed, "Cooperative access in cognitive radio networks: stable throughput and delay tradeoffs," Modeling and Optimization in Mobile, Ad Hoc and Wireless Networks (WiOpt), May 2014, http://arxiv.org/abs/1309.1200.

[10] M. Ashour, A. A. El-Sherif, T. ElBatt, and A. Mohamed, "Cognitive radio networks with probabilistic relaying: stable throughput and delay tradeoffs," http://arxiv.org/abs/1404.6112.

[11] R. M. Loynes, "The stability of a queue with non-independent interarrival and service times," Mathematical Proceedings of the Cambridge Philosophical Society, vol. 58, no. 3, pp. 497-520, Jul. 1962.

[12] M. Grant and S. Boyd, "CVX: Matlab software for disciplined convex programming, version 2.1," Mar. 2014, http://cvxr.com/cvx

[13] M. Grant and S. Boyd, "Graph implementations for nonsmooth convex programs," in Recent Advances in Learning and Control, V. Blondel, S. Boyd, and H. Kimura, Eds., Lecture Notes in Control and Information Sciences, pp. 95-110. Springer-Verlag Limited, 2008, http://stanford. edu/boyd/graph_dcp.html 